\title{
A re-appraisal of clinical and bacteriological criteria in the implementation of multiple drug therapy for leprosy control programmes and proposals for their better use
}

\author{
G D GEORGIEV* \& A C McDOUGALL† \\ *275 Turnpike Drive, Luton, Bedfordshire, LU3 3RD, England; \\ $\dagger 87$ Lower Radley, Near Abingdon, Oxfordshire, OX14 3BA, \\ England
}

Accepted for publication 27 October 1989

\section{Introduction}

The recommendations made by the World Health Organization (WHO) in 1982 for the treatment of all leprosy patients in control programmes with multiple drug regimens of relatively short duration ${ }^{1}$ have been implemented, to a varying extent, in most leprosyendemic countries. By the time of the XIIIth International Leprosy Congress in the Hague in $1988,{ }^{2}$ it was reported that over 2 million of the approximately 5 million registered cases of leprosy worldwide had been put on multiple drug therapy (MDT) and of those, over a quarter had completed their treatment and were no longer considered to have active disease. ${ }^{3}$ The proper implementation of MDT brings about a remarkable reduction in prevalence in a period of 5-10 years, accompanied by improvement in child and disability rates, and in the longer term, a reduction in incidence. ${ }^{4}$ Given such an effective tool for the control of leprosy, it is quite possible that its more determined use would reduce the worldwide problem of this disease by more than $75 \%$ by the year 2000 . Nevertheless, it is now clear that the rate and extent of implementation of MDT in some parts of the world, notably in the continent of Africa, is disconcertingly slow. It is therefore important to review the situation and try to identify factors which are impeding the implementation of a form of treatment which is not only available and affordable but highly effective. In previous publications, ${ }^{5,6}$ we expressed concern about the quality and standards of slit-skin smear bacteriological investigation in peripheral units of leprosy control programmes and called for a radical revision of the present approach. Our more recent experience from site visits to control programmes in Africa and South-east Asia, support the conclusion that the examination of slit-skin smears remains ' . . the weakest link in most leprosy control programmes. ${ }^{7,8}$

inherent in providing reliable laboratory services for slit-skin smear bacteriological examination in the field may, at least in some leprosy control programmes, be a deterrent to the expansion of MDT implementation. In a recent issue of Leprosy Review, Vettom \& Pritze described some of the problems which may be encountered in the important matter 
of quality control of smears from leprosy projects in different parts of the world. ${ }^{9} \mathrm{Nash}$ et al., writing from Papua New Guinea in the same issue, comment that '. . . we cannot afford to rely totally on laboratory services, which are often inaccessible or unreliable in developing countries', and they propose the use of a clinical 'leprosy score chart', as opposed to bacteriological criteria, for grouping patients for MDT in leprosy control programmes. ${ }^{10}$ There is little doubt that many national control programmes face serious difficulties in the establishment and maintenance of laboratory facilities for the proper examination of slit-skin smears in the field and it would be deplorable if this has to be rated as an unsolvable impediment to the implementation of MDT, especially if drugs, clinical expertise and other components of a potentially effective programme could be made available. Is there a possibility that this has indeed delayed the introduction of MDT in some parts of the world, thus depriving patients of a highly effective form of treatment? If this is the case, would it be reasonable to modify the present approach by proposing the use of well defined clinical, rather than strictly bacteriological criteria, in control programmes? In this communication we explore this question with particular attention to (a) the allocation of leprosy patients to paucibacillary (PB) or multibacillary (MB) groups, and (b) the determination of the end point (24 months or longer?) for chemotherapy in MB patients. Proposals are then made for operational changes which have the main objective of increasing the number of patients on MDT without unnecessary delay, whilst at the same time preserving reasonable standards of care and management.

\section{Allocation of patients to $\mathrm{PB}$ or MB regimens}

In 1982, the WHO publication Chemotherapy of leprosy for control programmes ${ }^{1}$ advised the allocation of all leprosy patients in need of specific treatment into either paucibacillary or multibacillary groups for the purpose of MDT. Paucibacillary leprosy included Indeterminate (I) and tuberculoid (T) leprosy in the Madrid classification, and I, TT and BT in the Ridley-Jopling classification with bacterial index (BI) $<2$ according to the Ridley scale at any site. Multibacillary leprosy included both lepromatous (L) and borderline (B) in the Madrid classification and polar lepromatous (LL), borderline lepromatous (BL) and mid-borderline (BB) leprosy in the Ridley-Jopling classification with $\mathrm{BI}$ of 2 or greater according to the Ridley scale at any site. ${ }^{1,11}$ The crucial importance of BI being less than 2 in PB cases was based on the understanding that these patients have a total bacillary load of less than $10^{6}$, with negligible risk of developing drug resistant mutants. This use of the $\mathrm{BI}$, for the grouping of patients for $\mathrm{PB}$ or $\mathrm{MB}$ regimens, has been followed by many control programmes implementing MDT since 1982 and probably widely interpreted as indicating that facilities for slit-skin smear examination are a prerequisite for its introduction.

National leprosy control programmes were required, at least ideally, to provide widely available and efficient laboratory services and assure uniform and reliable slit skin smear investigation in the field, including a system of quality control and continuous supervision. ${ }^{\prime}$ Yet a recent independent evaluation of one of the most developed and ambitious specialized leprosy control programmes ${ }^{7}$ has shown that $40 \%$ of the posts for laboratory technicians were vacant (in some places up to $83 \%$ ), $10 \%$ of those in position were not trained and only $40 \%$ of the registered patients had skin smear investigation. This situation is by no means exceptional. 
Despite the fact that the techniques for the taking, staining and reading of smears are not particularly demanding, there is continuing evidence that it is difficult to set up and maintain the necessary standards and uniformity. The reliability and validity of slit-skin smear BI examination in the field depends on experience in taking and fixing the smears as well as on strict adherence to the prescribed laboratory techniques-procedures often performed by different individuals (paramedical workers and laboratory technicians). The result is interpreted by a third person (medical officer or senior paramedical worker) who does not supervise the investigation but relies heavily on it in making his decisions. A lapse of quality at any stage of the procedure will render the results unreliable, including the possibility of false negative results. ${ }^{6}$ Vettom \& Pritze writing recently in this journal ${ }^{9}$ described a multicentre study on the reliability of slit-skin smears from control projects in different parts of the world. Correlation was judged to be satisfactory but the taking of smears was unsatisfactory in $26 \%$; staining unsatisfactory in $22 \%$ and reading unsatisfactory in $36 \%$ (Table 2 of the publication). Furthermore, in the 29 participating laboratories, 10 different staining methods for AFB were used (Table 3 of the publication) with wide variation in techniques. Even in well established laboratories there seems to be room for improvement in standardization and quality.

Before further reviewing the situation it should however be underlined that the BI examination is scientifically sound and valuable, provided the uniformity and reliability of the investigations can be assured. Leprosy institutions and projects which have reliable laboratory services should be encouraged to use it and further improve their services. However, the majority of leprosy control programmes do not have such facilities and standards. The question then arises: 'Is it feasible, with the existing infrastructure and resources, and within a reasonable period of time, to make reliable bacteriological investigation available to every patient needing MDT? And if this is not possible, what should be the course of action?' Perhaps the time has come to reconsider the present methodology of MDT implementation in control programmes and suggest an alternative operational strategy including the reorganization of laboratory services. The WHO Expert Committee on Leprosy (1988) defines 'a case of leprosy' as a 'person showing clinical signs of leprosy with or without bacteriological confirmation of the diagnosis . ...8 In the same Report it was concluded that there are clinical and operational reasons 'for including all smear-positive cases in the multibacillary group'. Therefore, a BI of 2 is no longer considered as the dividing point and the categorization of patients for MDT regimens is made simply on positive or negative bacteriological investigation of the slitskin smear. However, these developments do not solve the problems in control programmes where laboratory services remain inadequate and standards of bacterial examination low, and it is of interest that the Third Coordinating Meeting on Implementation on Multidrug Therapy concluded that there is a need for modification of current instructions in the method of MDT delivery with particular reference to 'noninsistence on slit-skin smear taken under certain situations'. ${ }^{12}$ In practice there is significant diversity in leprosy control programmes in the methods used to group patients for MDT. Some programmes group all clinically BB, BL and LL cases, whether skin smear positive or negative, as well as BT cases with 10 or more lesions (skin and nerve), as multibacillary. ${ }^{13}$ Others have opted to group patients on clinical grounds alone. ${ }^{14}$ It has been suggested that patients with disseminated multiple lesions with negative BI, when in doubt, should be treated as MB cases. ${ }^{15}$ In Papua New Guinea a 'score chart' to assist the clinical categorization of the patient is already in use. ${ }^{10}$ 
In order to avoid further confusion, it seems reasonable to consider alternatives to the bacteriological examination criteria for the grouping of the patients and the main purpose of this paper is to propose that the allocation of patients to $\mathrm{PB}$ and $\mathrm{MB}$ treatment regimens and a decision on the termination of the treatment in $\mathrm{MB}$ cases should be based, with very few exceptions, entirely on clinical criteria. This would clearly need the development of standardized and detailed guidelines for the instruction of staff in individual leprosy control programmes. Correlation between the clinical findings, immunological response and bacterial index has been well documented ${ }^{16}$ and the possibility that the number of lesions at the onset may be of practical value for the grouping of the patients for $\mathrm{PB}$ and $\mathrm{MB}$ regimens deserves further study. In this context it is of interest to note that a recent report by Deguerry et al. ${ }^{17}$ indicated that the number of macules (defined as all kinds of patches, either flat or partly or wholly elevated), may be a more important indicator of the probability of relapse, than regularity of treatment. A clinical system for grouping patients for MDT requires further attention, including the relation between the number of skin (or skin and nerve) lesions, classification and bacteriological positivity. It is intended to present data on this in a separate publication. ${ }^{18}$

In view of the proven value of lepromin testing, including the use of lepromin A, a s an aid to classification (but not diagnosis) its value in grouping patients, together with any other specific skin or serological test which may be developed, clearly needs consideration. On balance however, and in the control programme situation under consideration here, any additional benefit may be outweighed by the 3-week period before reading, the need to assure regular supplies of lepromin, syringes and needles, the training requirements for proper use and interpretation, and the possible risk of spreading hepatitis B and HIV infections. Even more importantly, the performance of a test which is incomprehensible to the average patient, together with the inherent 3-week delay, militates against the policy of 'presentation, diagnosis, allocation of regimen and treatment with MDT as soon as possible', which we would like to see established in all programmes which have appropriately trained staff.

By contrast, clinical examination, if completely and sympathetically carried out (using female staff where needed), is understandable to the average patient. By and large it is appreciated, and may improve contact and communication between health worker and patient whilst at the same time offering greater opportunities for the prevention, detection and treatment of disability due to nerve damage. The 'shift' of emphasis from a 'laboratory-dependant' approach to one which is clinically orientated may in fact be expected to strengthen the status of the patient as a participant, rather than a passive recipient, whilst also facilitating the wider and more rapid expansion of MDT to those who need it, provided the necessary orientation and training of staff can be achieved.

The use of the slit-skin smear technique, especially under field conditions, is a matter of considerable concern in relation to the possible spread of hepatitis B or human immunodeficiency (HIV) virus infection in either patients or health workers. The WHO has provided guidelines for the prevention of transmission during the performance of this procedure ${ }^{19,20}$ but those who have first hand experience of health services in developing countries may agree that they are far more likely to be put into practice in a supervised, central laboratory than in a peripheral laboratory or field unit.

\section{Multibacillary leprosy: 24 months treatment or longer?}

The WHO recommendations of $1982^{1}$ are for triple drug therapy (rifampicin, clofazimine, 
dapsone) in multibacillary cases for a minimum of 2 years, but whenever possible, up to smear negativity. Since some MB cases have a BI of 6 at the onset and since it drops (regardless of the type of treatment used) by $0 \cdot 6-1 \log$ per year ${ }^{21}$ it is quite possible that MDT would have to continue for several years after the completion of 24 doses of treatment, if smear negativity is chosen as the end point. Apart from the fact that this could almost triple the total cost of treatment in many patients, its adoption as a general policy would be operationally difficult to maintain and would undermine one of the most important attractions of MDT as being defined and of relatively short duration. A recent workshop organized by the Armauer Hansen Institute in Germany and attended by leading scientists in the leprosy field, reviewed in detail the duration of MDT and the criteria for the termination of treatment in MB cases. ${ }^{22}$ It was evident that neither skin smear BI evaluation, histopathology, immunological tests nor an assessment of clinical progress can alone indicate the point at which to stop treatment in the individual patient. Clinical improvement during treatment, especially in active and previously untreated cases in fact occurs much earlier than any move in the BI scale, often earlier with MDT than with dapsone monotherapy. Studies in Malta have shown that $20 \cdot 7 \%$ of treated MB patients remain bacteriologically positive for 5 to 12 years without relapsing. ${ }^{23}$ Furthermore, neither the regimen used, nor its duration appear to have any effect on microbial persistence. ${ }^{24}$

From an operational point of view the precise time taken to 'cure' a patient, especially if the 'end-point' is based on bacteriological findings, is far less important than the average duration of treatment needed to prevent disability (other than that which was established at the onset) and produce an acceptably low relapse rate after treatment. In practice many control programmes have elected to routinely stop MDT in MB cases at 24 months (or 24 doses in 36 months) irrespective of bacteriological positivity, and the results, in the periods of observation so far, are extremely encouraging with regard to the low rate of relapse. Reports of rifampicin resistance, recently published, ${ }^{25}$ underline the importance of regular monthly attendance for supervised medication, whilst at the same time doing all possible to ensure adherence to the prescribed daily domiciliary intake of dapsone and clofazimine by the patient. But experience to date from many control programmes seems to indicate that a 24-month period of regular triple therapy for MB patients is satisfactory. Is there any good reason for the performance of routine slit-skin smear at this point or subsequently?

\section{Proposals}

As a basis for discussion, but with emphasis on changes which can be made with the minimum of delay, we propose the following:

\section{1 'Routine' smears}

The 'routine' (often meaningless) use of slit-skin smears in control programmes should be stopped. Their use should be reserved for selected cases, with specific indications decided by control programmes and outlined in their guidelines.

\section{Peripheral 'one-man' laboratories}

Peripheral, unsupervised, 'one-man' units should be scaled down and in most cases phased out altogether. 


\section{A central or reference laboratory}

Either as part of a general laboratory, or as a sub-unit, one central or reference laboratory of high quality should be available at district, provincial or central level, depending on the health services and the leprosy control programme structure, for the examination of smears under the best conditions.

\section{Smear taking and selection of sites}

In view of the crucial importance of the selection of sites and the taking of smears, short, intensive re-training courses, supported by written and illustrated instructions (in appropriate languages) should be arranged for all those who will be entrusted with this procedure. This must include precise instructions on fixation, labelling of slides, recordkeeping and despatch of slides to the central or reference laboratory for staining and interpretation. A similar short module for the taking of smears (not the selection of sites) should be arranged for senior staff in reference laboratories. This will necessitate an efficient system for the despatch of reports back to the control units with the minimum of delay. These measures should help to achieve acceptable and comparable standards within and between programmes.

\section{Hepatitis B and AIDS virus}

The possible risks should be taken seriously. All staff involved in handling the slit-skin smears should be aware of the possible risks of viral infection from hepatitis B or AIDS and be familiar with the guidelines for protection issued by WHO. ${ }^{15,16}$

\section{Allocation of patients to pauci- or multi-bacillary regimens}

In principle this should be made on the basis of clinical criteria only, except in selected, doubtful cases. Guidelines on how to do this, training modules for all grades of field staff and appropriate written and illustrated material (in local languages) all require expert input and production. This process also calls for a built-in system to enable senior staff to check the accuracy of allocation in control programmes, together with careful monitoring of clinical outcome (see 8 below).

\section{The duration of MDT for multi-bacillary cases}

Rather than continuing to smear negativity (at all sites), a period of 24 months triple drug therapy should be accepted as adequate. Unless in exceptional doubtful cases, smears should not be routinely taken at this point or subsequently. The decision to stop treatment at 24 months will thus be based on (a) the record of regular attendance for 24 supervised months doses, (b) any available evidence that compliance to unsupervised daily medication has been satisfactory, and (c) the clinical findings.

\section{Diagnosis of relapses}

Relapse rate is perhaps the most reliable criterium for the assessment of the effectiveness 
of treatment and control measures. However, the diagnosis of relapse is not always easy, particularly in paucibacillary cases. It is strongly recommended that suspected relapse cases should be thoroughly investigated in referral centres before a diagnosis of relapse is accepted.

\section{Technical support}

At regional/district level, particularly in integrated programmes, it is essential to provide and maintain clinical expertise for complications and difficult cases alongside laboratory services, for the guidance and support of paramedical workers.

\section{Operational research}

The above changes call for careful prospective planning and the monitoring (and rapid solving) of problems which may arise during their implementation. The proposals in paragraph 6 above, call for expert advice and it would be helpful if this and the other operational changes described in this paper could be addressed by an expert committee or group, with attention to a plan of action, funding, training modules, supervision, monitoring and research. The latter should include retrospective studies from well-run programmes on the relationship between (a) the number of skin lesions and (b) the number of skin and nerve lesions on the one hand, and the classification (TT, BT, I etc) as used in the current MDT grouping on the other. These results should in turn be analysed in relation to eventual clinical outcome and relapse rates. Comparative studies, in terms of cure and prevention of disability in patients allocated to regimens on clinical grounds alone, as opposed to those allocated on combined clinical and bacteriological grounds, could also be carried out. However, neither these, nor any of the studies mentioned above, should be regarded as a reason to delay the implementation of the operational changes proposed in this paper.

\section{Summary and conclusions}

Behind the appraisal of criteria above and our proposals, is the question 'To what extent and for what purpose should slit-skin smears be used in programmes implementing MDT?' The answer may lie somewhere between the extreme of abolishing them altogether and the present situation, in which many people seem to think that a comprehensive service has to be provided at many levels, including the periphery, almost regardless of quality and with minimal supervision.

Our plea is twofold: (1) the much wider use of standardized clinical, as opposed to bacteriological, criteria for the grouping of the patients, the termination of treatment in multibacillary cases and follow up, and (2) the abolition of the routine use of slit-skin smear examination in the field, coupled with the phasing out of unsupervised peripheral units of doubtful quality and the identification of one laboratory at central or provincial level which is able to provide a reliable, high quality service for smears in selected cases. Clearly these proposals will not solve all the operational and methodological problems. There is an urgent need for research on the best ways to implement MDT on a large scale. But the truth of the matter is that 7 years after the WHO recommendations ${ }^{1}$ and for 
reasons which have so far been poorly defined, less than half the world's registered cases have so far received MDT. Most of the registered cases continue to take a form of treatment, dapsone monotherapy, which was condemned over 10 years ago as being hazardous on account of the dangers of resistance. From the estimated world total of 10 12 million cases, WHO has suggested that as many as 8 million cases may still be in need of MDT. ${ }^{3}$ If we are trying to move towards a goal of 'MDT for all by the year 2000', there may still be a long way to go. Operational problems are experienced in every control programme and there is an urgent need to find the right solutions through well coordinated operational research. Would it be an exaggeration to say that the current priority in leprosy control is the establishment of a permanent international committee to identify, as rapidly as possible, the main factors which are impeding the implementation of MDT, so that realistic solutions may be found?

\section{References}

1 WHO Study Group. Chemotherapy of leprosy for control programmes. Technical Report Series 675. WHO: Geneva, 1982.

2 Transactions of the XIIIth International Leprosy Congress, The Hague, September 1988. Int J Lepr, 1989; 57: $231-44$.

3 WHO. Multidrug therapy for leprosy; an end in sight. WHO: Geneva, 1988.

4 UNDP/World Bank/WHO. Report of Progress 1987-1989. Special Programme for Research and Training in Tropical Diseases (TDR). WHO: Geneva, 1989.

5 Georgiev GD, McDougall AC. Skin Smears and the Bacterial Index (BI) in Multiple Drug Therapy Leprosy Control Programs: An Unsatisfactory and Potentially Hazardous State of Affairs. Correspondence. Int $J$ Lepr, 1988; 56: 101-4.

${ }^{6}$ Georgiev GD, McDougall AC. The bacteriological examination of slit-skin smear in leprosy control programmes using multiple drug therapy: A plea for radical changes in current operational methodology. Ind J Lepr, 1987; 59: 373-86.

7 National Leprosy Eradication Programme in India. Report of Second Independent Evaluation. Ministry of Health and Family Welfare, Nirman Bhawan, New Delhi, 1987.

8 WHO Expert Committee on Leprosy. Sixth Report. Technical Report Series 768. WHO: Geneva, 1988.

9 Vettom L, Pritze S. Reliability of skin smear results: experience with quality control of skin smears in different routine services in leprosy control programmes. Lepr Rev, 1989; 60: 187-97.

10 Nash JE, Hudson BJ, Pyakalyia T. Leprosy score chart to assist classification. Letter to the Editor. Lepr Rev, 1989; 60: 3, 242-3.

11 WHO Study Group. Epidemiology of leprosy in relation to control. Technical Report Series 716. WHO: Geneva, 1985.

12 WHO. Report of third coordinating meeting on implementation of multidrug therapy (MDT) in leprosy control programmes. WHO/CDS/LEP/88.4. WHO: Geneva, 1988.

13 Guidelines for multidrug treatment in endemic districts. National leprosy eradication programme. Directorate General of Health Services, Ministry of Health and Family Welfare (1989), Nirwan Bhawan, New Delhi.

14 WHO. Second Co-ordinating Meeting on Implementation of Multidrug Therapy in Leprosy Control. WHO: Geneva, 1986.

15 WHO. Report of a consultation on implementation of multidrug therapy for leprosy control. WHO/LEPR/ 85.1. WHO: Geneva, 1985.

16 Meyers WM et al. The Ridley-Jopling five-group classification of leprosy-correlation of parameters of the classification in 1429 leprosy patients. Int J Le pr, 1979; 47: 683-4.

17 Deguerry M, Declercq E, Misson C, Vellut C, Bertrand F. Registration of the number of macules in paucibacillary leprosy for evaluation of early diagnosis and individual prognosis. Lepr Rev, 1989; 60:206-13.

18 McDougall AC, Georgiev GD. Multiple drug therapy for leprosy control: relationship between the number of lesions, the bacterial index (BI) and the grouping of cases for pauci- or multi-bacillary treatment regimens. In preparation.

19 WHO. Guidelines for personnel involved in collection of skin smears in leprosy control programmes for prevention and control of possible infection with HIV. WHO: Geneva, 1987. 
20 WHO. Guidelines on sterilization and high-level disinfection methods effective against human immunodeficiency virus (HIV). WHO AIDS Series, No. 2, 1988.

${ }^{21}$ Grosset JH. Recent developments in the field of multidrug therapy and future research in chemotherapy of leprosy. Lepr Rev, 1986; 57: (suppl. 3), 223-34.

22 Pritze S. (Edit) Workshop 'Criteria To Determine The Exact End of Multidrug Therapy in Leprosy'. Armauer Hansen Institute. Dominikanerplatz 4, PO Box 348, Wurzburg, W. Germany.

23 Jopling WH. A report on two follow-up investigations of the Malta-Project, 1983 and 1986. Lepr Rev, 1986; 57: suppl 3, 47-53.

24 THELEP. Subcommittee on Clinical Trials of the Chemotherapy of Leprosy (THELEP). Scientific Working Group of the UNDP/World Bank/WHO Special Programme for Research and Training in Tropical Diseases. Persisting Mycobacterium leprae among THELEP trial patients in Bamako and Chingleput. Lepr Rev, 1987; 58: 325-37.

25 Grosset JH, Guelpa-Lauras CC, Bobin P, Brucker G, Cartel JL, Constant-Desportes M, Falguel B, Frederic M, Guillaume JC, Millan J. Study of 39 documented relapses of multibacillary leprosy after treatment with rifampin. Int J Lepr, 1989; 57: 607-14. 